\title{
Flow Sensors - Recent and Future Developments
}

\author{
Becker, Stefan \\ University Erlangen-Nuremberg \\ Cauerstr. 4, 90158 Erlangen
}

\section{Abstract}

The objective of the presentation is to provide a comprehensive overview of recent developments concerning sensors that can be used to measure the flow velocity. Velocity measurements can be understood to represent spatially integrated and pointwise values. The current overview will be focussed on pointwise measurement techniques including sensor developments in the field of thermal anemometers, pressure-based velocity sensors and laser measurement techniques.

\section{Introduction}

The application of flow sensors is closely associated with the objectives of the measurements and the test conditions. It is worth to make a distinction between the measuring variable determinating, the measuring principle and the resolution of the measurement which contains the temporal and spatial scales. Fig. 1 gives an overview of the different areas in the current measuring technology.

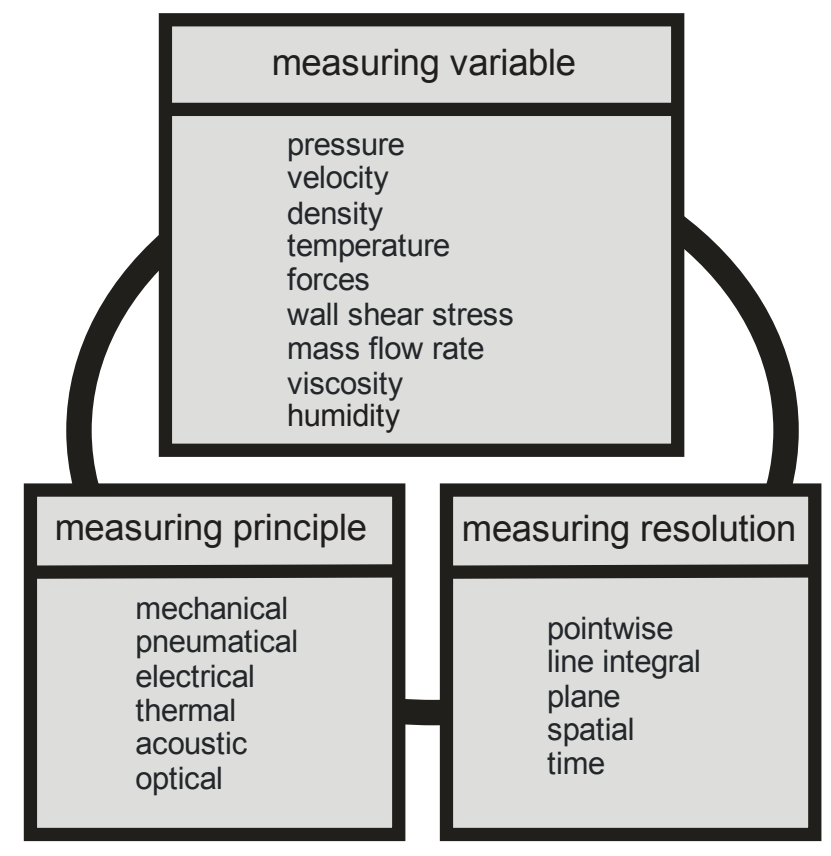

Fig. 1: Different areas in the current measuring technology

Important I in the application of flow sensors are the measured variables. They define beside the measuring variables the necessary spatial and temporal resolution of the measurement. Based on these criteria, together with spatial and temporal boundary conditions, the selection of the measuring flow sensors occurs. Basic information to the inclusion of such considerations in the definition of certain measuring procedures are found in books by Wuest [1], Eckelmann [2], Fiedler [3] and Nitsche [4],[5]. The application of the measuring procedures in turbulent flows describes Bradshaw in [6].

The current paper is focussed on the application of flow sensors in isothermal, incompressible, newtonian fluids in which the flow field is described by the pressure and velocity distribution. The capture of the local pressure occurs with priority by using pressure probes. For the measurement of velocity distribution beside the pressure probes thermal-electric sensors (hot wire anemometry-HWA), optical methods (e.g. 
laser Doppler anemomety - LDA, Particle image Velocimetry - PIV) and acoustic sensors (ultrasonic sensors) are available.

\section{Pressure probes}

The measuring technology belongs to the classical local measuring procedures by using pressure probes. On this occasion should be distinguished between the pressure probes, which directly are introduced into the flowfield, and the probes measuring the wall press which are attached in the wall surface. The probes directly introduced into the flow, are classical Pitot-and Prandtl probes. A lot of investigations in the literature has shown, that the mechanical disturbance effect causeby the diameter of the probe body on the measuring result is low and hence, can be neglected. The influence of the flow angle of attack on the measuring result is substantially more important. It is pointed out, that already by very small changes of the flow angle around 5 degree, the divergences in the measurement of the whole pressure are not neglectable. With smaller growing probe diameter relation (inside diameter to external diameter) the sensitivity of the probe increases. Improvements are achieved by modifications in the probe head. An additional pipe around the probe head (e.g., in the form of a Venturi nozzle) allows measurements with a variation in the angle of attack to \pm 40 degree too. However, it comes to an enlargement of the probe diameter, and what entails a reduction of the spatial resolution.

For the capture of the direction of the velocity vector improvements become achieved by the application of multi hole probes. The measured pressure difference between each pressure hole of the probe is valid as a measure of the determination of the angle of attack. Especially with investigations in wall boundary layers two or three pressure measuring holes in probe head are sufficient for the measurements of the angle of attack. The determination of the angle of attack and the velocity vector requires in the approach the production of a calibration curve. The calibration curve contains for given angle of attack the pressure difference of both lateral pressure holes. The allowed region of the angle of attack imits itself to approx. \pm 25 degree around the main flow direction.

Other improvements to the capture of the spatial orientation of the velocity vectors require the application of four or five hole probes. The direction of velocity vector to the measuring probe is defined by the angles of attack and yaw. Beside the local pressure and the total pressure four independent measuring variables are to determine. The flow-mechanical basic principle for the probe head geometry and the matching measuring algorithm provide the potential theory of flow around a sphere. More information in applications of four hole probes is given in [1] and [7]. The figure 2 shows a four hole pressure probes rack.

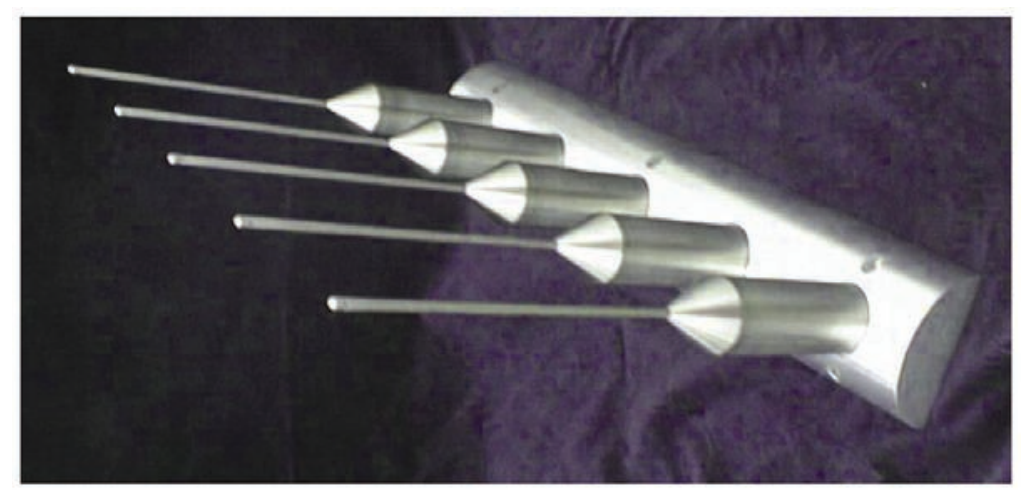

Fig. 2 Rack of four hole pressure probes

This probe configuration was developed for the application of boundary layer measurements in very thick boundary layers. Those probes are applied for investigations in thick boundray layers under rough measuring conditions for example for measurements of the wall boundary layers on trains.

Extensions and improvements to detect flow vectors with varying velocity directions have been achieved by using fourteen hole probes. The head of the fourteen hole probe is composed of two seven hole probes (see fig. 3). With a suitable shutter algorithm based on the momentary flow direction it is an aim to activate the suitable probe head half for the measurement. In the foreground of the applications rise e.g. flow investigations in the recirculation flow region behind cars. Additional to the the velocity vector the probe allowes to determine the vorticity field.( see [8]) 

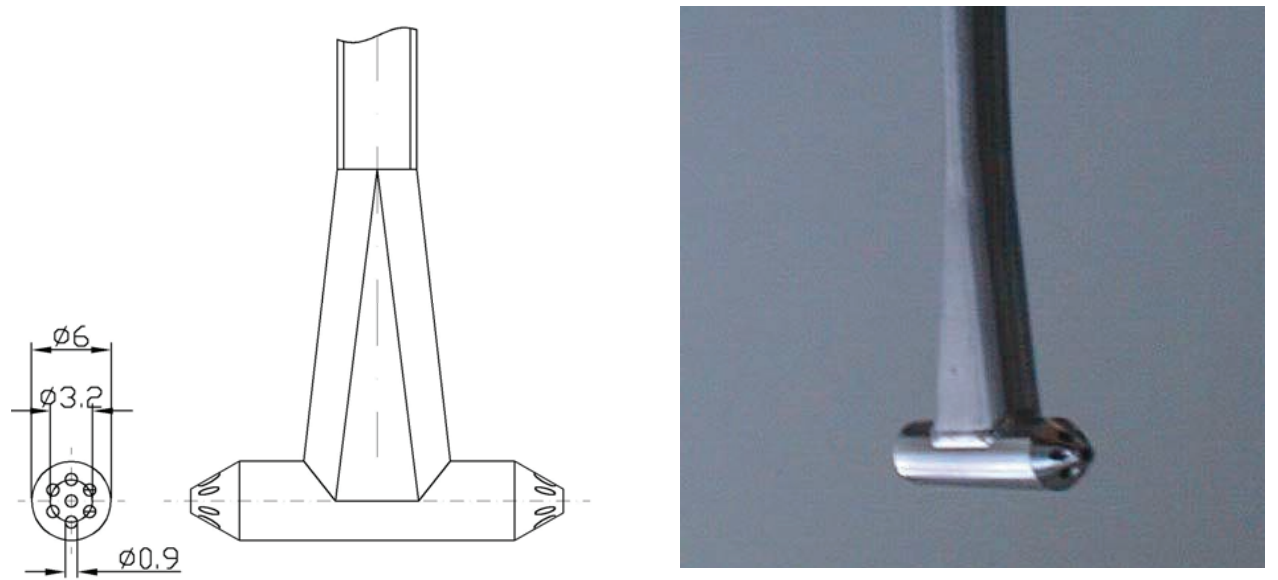

Fig. 3: Fourteen hole pressure probe

\section{Thermal-electric sensors}

The use of the hot wire sensors for the investigation of the flow field belongs to the established measuring methods to the capture local velocity distribution. The time depending averaged velocity, values of the Reynolds stress tensor and other moments higher order are able to determine with a very high temporal resolution. Extensively the work with hot wire probe is described in [9]. Overview articles to the measuring principle are found among other things in [1], [2], [3] and [5]. The use of the measuring procedure for investigations in turbulent flows was done in [6].

The design of the hot-wire permits a differentiation between hot-wire and hot films. Hot films exist in contrast to the hot-wire of a metal-coated glass cylinder whose diameter lies with approx. $d \approx 50 \mu \mathrm{m}$. Thereby a higher mechanical robustness of the sensor element becomes guaranteed. Nevertheless, with it reduces the sensitivity of the sensor element. This means, that only a limited region of the turbulent velocity fluctuations can be dissolved. For wall bounded flows hot-wire probes become priority for the application. Exceptions if strongly dirty or particle-loaded flows, because form here the particle to demages of the thin hot-wire sensor lead. Other areas of application of the hot films are, e.g., water flow and flows with higher viscosity, with which the frequency of the velocity fluctuations are relatively small.

According to the number of the hot-wires in the sensor element there are one-wire, two-wire and multi-wire probes. Fig. 4 shows schematically their basic construction. In the use one-wire probes permit no direct regulation of the direction of the flow vector. With two-wire probes or in their mplementation as a x-wire probe a two-dimensional determination of the direction of the velocity vector is possible.

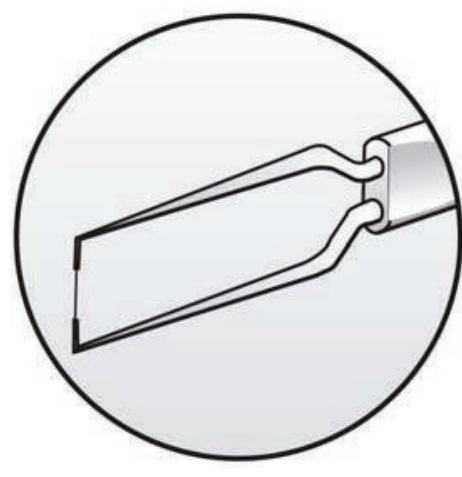

a)

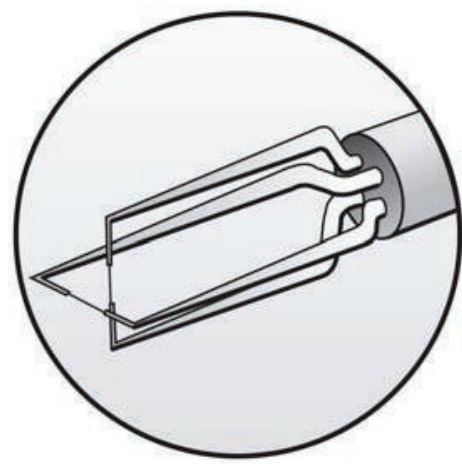

b)

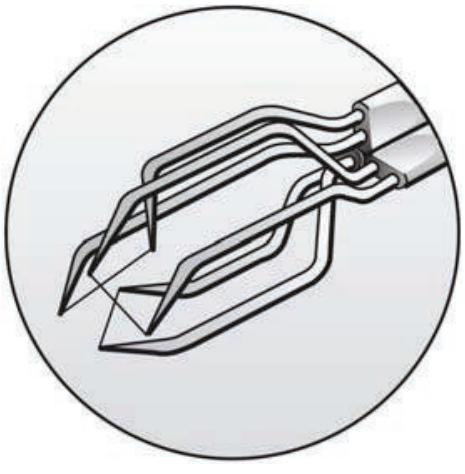

c)

Fig. 4 a) one-wire probe, b) $x$-wire probe and c) three-wire probe 
One of the current developments is the application of suitable correction procedures for the investigations of flow in wall nearness. Sources of influence are the wall material, the overheating ration, the probe shape (e.g. probe length, probe configuration) see fig. 5. On account of the whole complexity of the problem itself it is not possible to formulate an universal correction procedure up to now.

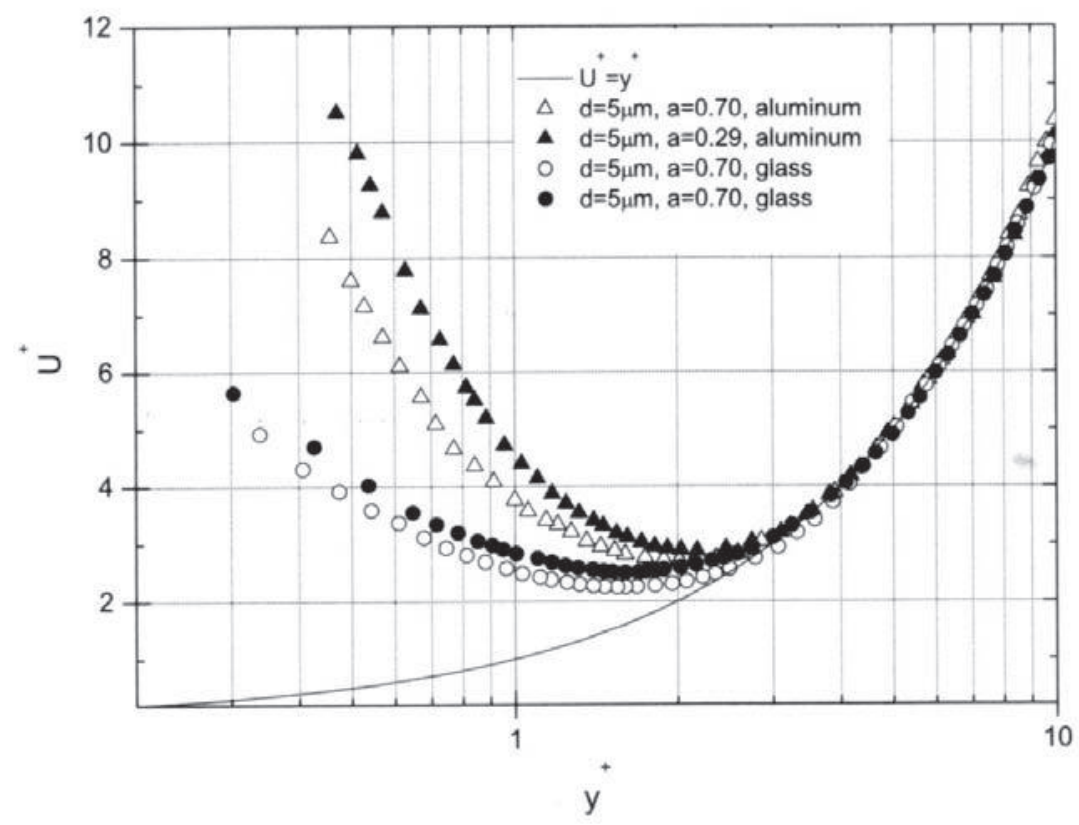

Fig. 5: Hot-wire measurements closed to the wall by different wall materials [10]

\section{Optical sensor}

Most of used optical flow sensors are laser Doppler anemometry sensors or particle image velocimetry systems. All laser based velocity measurements are particle based techniques, e.g. laser Doppler anemometry (LDA), particle image velocimetry (PIV), Doppler global velocimetry (DGV) and laser transit velocimetry (LTV). These velocity sensors rely on the presence of tracer or seeding particles in the flow that not only follow all velocity fluctuations but are also sufficient in number providing the desired spatial and temporal resolution of the flow field. Suitable particles are still an open problem, because it is desirable that these tracers are at the same time good light-scattering centers, since this improves the signal quality for a given incident laser power. The fact that flow tracking demands small particle diameters while light scattering improves with increasing particles size already suggests the requirement for optimization when selecting seeding particles. The best way would be to avoid an additional particle seeding by only using the natural particle distribution in the flow.

Fig. 6 shows some recent developments concerning laser based flow sensor which allow measurements without any additional particle seeding in air flows. The probe was developed for inflight flight measurements on an air plane wings. The system development yielding a miniaturized optical sensor system optimized to be small, light and robust and to be able to detect signals from very small scattering particles. Fig. 7 gives impressions about typically results from the in-flight measurements. The mean streamwise velocities, turbulent velocity fluctuations and energy spectra of the fluctuating components were measured. The results presented demonstrate the successful complementation of the development. The LDA-probe is available for aerodynamic studies under in-flight condition. In particular it can be a powerful tool for laminar wing research. There is also still room for further developments and related work. Whereas the optical system design dominated in the present research efforts, conventional LDA signal processing equipment was employed. Future work should concentrate on developing LDA electronic systems that are very small in size, light in weight and robust, so that they can be integrated in the probe design. It should also aim for a reduction in power consumption of the electronic system to permit long time measurements during in-flights. 

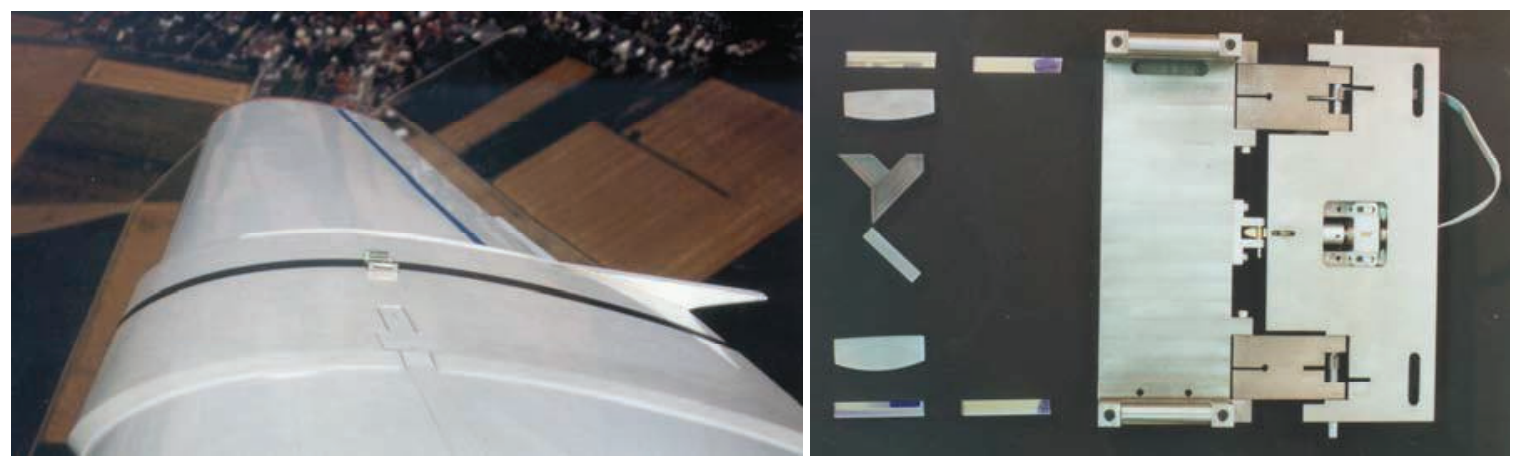

Fig. 6: LDA-probe during in-flight test on an airplane wing and probe design
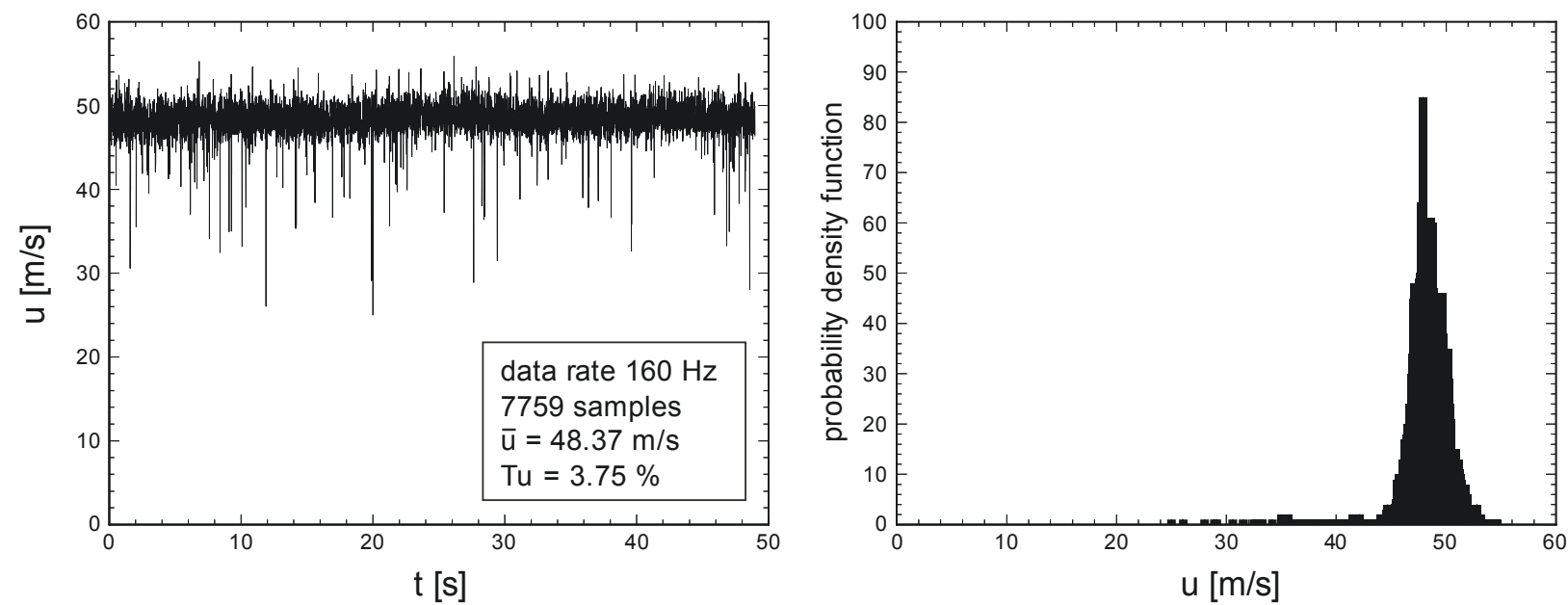

Fig. 7: Typical time series of instantaneous velocity and PDF during inflight measurements

In principle the spatial resolution of LDA system is determined by the size of the measuring volume. One should take care of the spatial average effect caused by the finite size of the measurement volume. The sensor cannot resolve fluctuations with a scale smaller than the size of the measuring volume and they are spatially averaged. It is possible to reduce the detection volume by employing a spatial mask together with side-scatter detection, which nescessitates two optical access to the flow. A higher spatial resolution is expected by reducing the size of the measuring volume. Strong focussing of laser beams achieves smaller size of measuring volume. However,this causes non-uniformity of fringe spacings inside the measuring volume and smaller numbers of fringes, which lower the accuracy of the velocity measurements and induce apparent turbulence intensities.

The LDA velocity-profile sensor by Czarske [12], Shirai [13],[14] has been proposed to overcome the spatial resolution problem. The principle of the optical profil sensor is shown in fig. 7.

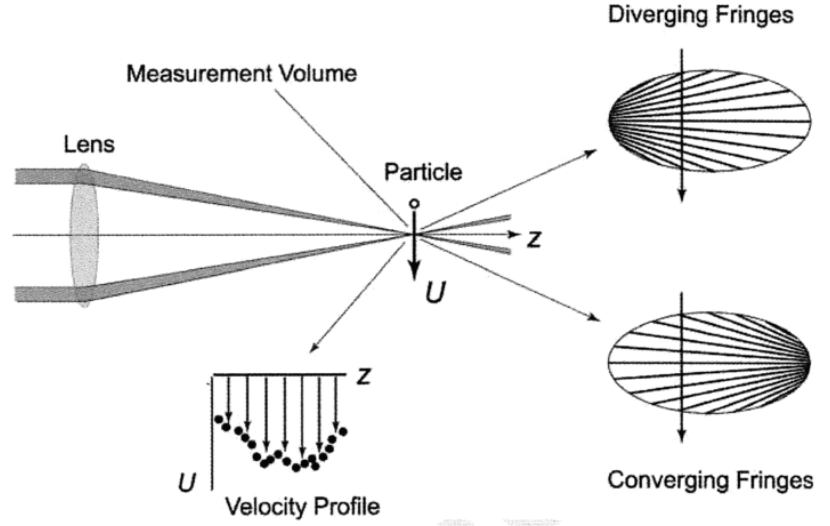

Fig. 8: Principle of the velocity profile sensor. [13] 
The use of two fringe systems allow the determination of the positions as well as the velocities of single tracer particles in the measuring volume. The relative accuracy was estimated to be $0.1 \%$. The sensor was successfully tested in several laminar and turbulent shear layers [13]. Fig. 9. shows as an example results of the measurements in a full developed turbulent channel flow. The measured mean velocities are in good agreement with theoretical data.

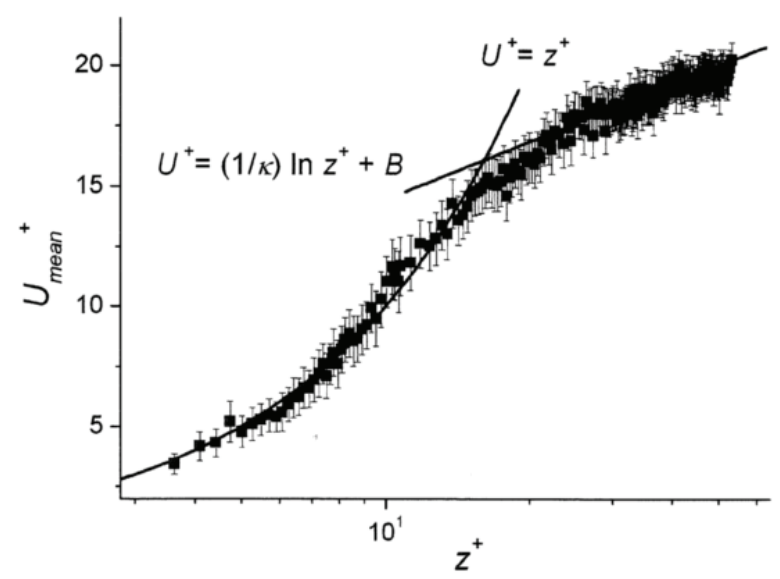

Fig. 9: Mean velocity profile near the wall with measurement uncertainties plottet as error bars [12]

\section{Conclusions}

The paper gives an overview of current and new developments to measure pointwise pressure and velocity distribution in the flow field. Most of the applications were done in the direction to improve the spatial and temporal resolution of the measurements. Compact and robust sensors were developed to use special sensors under free field conditions on trains, cars and airplanes. Future developments have to go in direction to miniaturized low power flow sensors in which the electronical components are embedded in flow probe.

7. Literature

[1] W. Wuest. Strömungsmesstechnik. Vieweg-Verlag, Braunschweig, 1997.

[2] H. Eckelmann. Einführung in die Strömungsmesstechnik. B.G. Teubner Verlag, Stuttgart, 1997.

[3] Ó. Fiedler. Strömungs und Durchflußmeßtechnik, R. Oldenbourg Verlag, 1992

[4] Nitsche. Strömungsmesstechnik. Springer Verlag, Berlin, Heidelberg, New York, 1 edition, 1994.

[5] W. Nitsche and A. Brunn. Strömungsmesstechnik. Springer Verlag, Berlin, Heidelberg, New York, 2 edition, 2005.

[6] P. Bradshaw. An Introduction to Turbulence and its Measurement. Pergamon Press, Oxford-New York-Toronto-Sydney-Braunschweig, 1st edition, 1971.

[7] R.T. Müller, H. Lienhart, and G. Schwarz. Four-hole directional probe for measurements in incompressible flows. Zeitschrift fuer Flugwissenschaften und Weltraumforschung 9:370-375, 1985.

[8] H. Lienhart. Vierzehnlochsonde. Technical report, LSTM-Erlangen, 2001.

[9] H.H. Bruun. Hot-Wire Anemometry, Oxford Science Publications, 1995

[10] E.S. Zanoun and H. Durst, F.and Nagib. Evaluating the law of the wall in two dimensional fully developed turbulent channel flow. Phys. Fluids, 15:1-11, 2003.

[11] J. Czarske. Laser Doppler velocity profile sensor using a chromatic coding, Meas. Sc. Technol. 12: 52-57, 2001

[12] K. Shirai , T. Pfister, L. Büttner, J. Czarske, H. Müller, S. Becker, H. Lienhart, H. and F. Durst. Highly spatially resolved velocity measurements of a turbulent channel flow by a fiber-optic heterodyne laser-Doppler velocity-profile sensor. Experiments in Fluids, Springer Berlin / Heidelberg, Vol. 40, No. 3, pp. 473 - 481;2006.

[13] K.Shirai. Investigation and Application of Laser Doppler Velocity Profile Sensors toward Measurements of Turbulent Shear Flows, Dissertation Tu Dresden, Shaker Verlag, Aachen 2010 